\title{
First principle calculation of structural, electronic and elastic properties of rare earth nitride
}

\author{
Purvee BhardWaJ*, SAdHNA Singh
}

High Pressure Research Lab., Department of Physics, Barkatullah University, Bhopal-462026, India

\begin{abstract}
First principle calculation of the electronic and elastic properties of $\mathrm{CeN}$ nitride, which crystallizes in the rock-salt structure, is reported in the present paper. The ground state properties, such as lattice constant $\left(\mathrm{a}_{0}\right)$, bulk modulus (B) and its pressure derivative $\left(\mathrm{B}^{\prime}\right)$ are reported. These results show good agreement with the experimental and other theoretical results. Besides, we have studied the Murnaghan's equation of state, and used it to fit the theoretical electronic ground state energy and obtain thermodynamic quantities such as the bulk modulus. Furthermore the electronic band structure, total density of states and partial density of states of $\mathrm{CeN}$ are also discussed.
\end{abstract}

Keywords: nitride; electronic properties; band structure; elastic properties

(C) Wroclaw University of Technology.

\section{Introduction}

Among rare earth compounds nitrides of rareearth are an interesting group of materials combining theoretical and experimental challenges. These rare earth nitrides (RENs) attract attention in the extensive technological applications. These materials show a strong coupling between their magnetic and electronic properties. The members of RENs series demonstrate a range of electronic and magnetic properties. The RENs are used in spintronics. The property of spin is used in electronic devices to "store, encode, access, process and transmit information." The rare earth nitrides lie on the boundary between metals and insulators. The RENs materials have high magnetic moment and form a wide range of magnetic structures. Due to this, they are strong candidates for applications in spintronics and spinfiltering devices. The RENs posses partially filled f-electron orbital. All the RENs crystallize into the $\mathrm{NaCl}$ rock salt structure at normal temperature and pressure [1-4].

The first compound of this series is cerium nitride $(\mathrm{CeN})$. The chemistry of rare earth elements

*E-mail: purveebhardwaj@gmail.com is largely determined by the ionic radius, which decreases steadily along with the series corresponding to the fillings of the $4 \mathrm{f}$-orbitals. $\mathrm{CeN}$ has some unusual properties compared with the other rare-earth nitrides. It shows mixed valence behavior. The pressure induced structural phase transition of $\mathrm{CeN}$ is an interesting topic of research. Very little theoretical or experimental work has been reported on the structural and electronic properties of CeN. Danan et al. [5] studied the temperature dependence of lattice constant and magnetic susceptibility of CeN. Bulk calculations of $\mathrm{CeN}$ have been performed by Svane et al. [6]. The structural and elastic properties under high pressure of $\mathrm{CeN}$ have been investigated using two-body potential theory with ionic modified charge by Rukmangad et al. [7]. Epitaxial layers of $\mathrm{CeN}$ on $\mathrm{Mg}\left(\begin{array}{lll}0 & 0 & 1\end{array}\right)$ have recently been investigated by Lee et al. [8]. Kanchana et al. [9] published a theoretical work on the lattice dynamics and elastic properties of $\mathrm{CeN}$ using ab initio density-functional methods. Both the local density approximation (LDA) and the generalized gradient approximation (GGA) were used for the exchange-correlation potential. The high-pressure structural stability of $\mathrm{CeN}$ is investigated by both experiment and theory [10]. Experiments are carried out by energy-dispersive X-ray 
diffraction and synchrotron radiation, using a diamond anvil cell, to a maximum pressure of $77 \mathrm{GPa}$.

According to the above reported data $\mathrm{CeN}$ has been less studied in the literature. Previous calculations presented the results on lattice dynamics, magnetic, elastic and bulk properties of CeN. These theoretical calculations were based on either ab-initio calculations or two-body potential model mainly, while in the present study we have used density functional theory with the generalized gradient approximation within the treatment of $4 \mathrm{f}$-states. We have used plane wave pseudo potential density functional theory as implemented in the quantum espresso code [11]. The ground state properties of B1-type CeN have been studied using this approach. The lattice constant and bulk modulus of this rare earth nitride have also been reported. We further report the electronic band structure (BS) and density of states (DOS) for CeN. The organization of this article is as follows: in Section 2 the method of computation of the quantum espresso calculation is described. In Section 3, the results with some predictions are discussed. The corresponding Sections 3.1, 3.2 and 3.3 deal with the structural, elastic and electronic properties of $\mathrm{CeN}$. Finally, in Section 4, we have concluded the results.

\section{The method of first principle calculation}

Quantum Espresso is mainly based on DFT theory (electron-ion interaction), plane wave and pseudopotentials (electron-electron interaction). It calculates the ground state energy and Kohn Sham orbital for various types of structural optimizations. Three main components of the method are PWSCF (plane wave self consistent field), CP (Car-Parinello), FPMD (first principle molecular dynamics). For quantum espresso, irreducible kpoints were generated according to the MonkhorstPack scheme [12]. The Kohn-Sham single-particle functions were expanded on a basis of plane-wave set with a kinetic energy cut-off of $36 \mathrm{Ry}$. Brillouinzone was sampled with $12 \times 12 \times 12 \mathrm{k}$-point mesh in order to get well converged ground state energy. The cerium nitride $\mathrm{CeN}$ considered in the present work crystallizes in NaCl-type (Fm3m, 22 5) structure at ambient conditions. The cerium atom is positioned at $(0,0,0)$ and nitride at $(1 / 2,1 / 2,1 / 2)$. Under pressure it transforms to CsCl-type ( $\mathrm{Pm} 3 \mathrm{~m}$, 22 1) structure in which the nitride is positioned at $(1 / 2,1 / 2,1 / 2)$. The exchange and correlation effects have been treated within the GGA. The lattice parameter, bulk modulus and pressure derivative of the bulk modulus were determined by the standard procedure of computing the total energy for different volumes and fitted to Murnaghan's equation of state [13].

\section{Result and discussion}

\subsection{Ground state properties}

For ground state properties of narrow gap semiconductors the total energies were calculated as a function of lattice constant in $\mathrm{NaCl}$-type structure using the first principles pseudopotential (PWSCF) method. The equilibrium lattice constants were obtained by minimizing the total energy and these parameters were used in the non self-consistent calculations of the band structure. For Quantum Espresso these energy values have been fitted to the Murnaghan's equation of state [13] to obtain the equilibrium lattice constant (a), bulk modulus (B) and its pressure derivative $\left(\mathrm{B}^{\prime}\right)$ at minimum equilibrium volume $\mathrm{V}_{0}$ :

$$
P(V)=\frac{B}{B^{\prime}}\left[\left(\frac{V_{0}}{V}\right)^{B^{\prime}}-1\right]
$$

where the fit parameters are the equilibrium volume $\mathrm{V}_{0}$ and the bulk modulus $\mathrm{B}$ :

$$
B=-V \frac{\partial P}{\partial V}=V \frac{\partial^{2} E}{\partial V^{2}}
$$

and its derivative with respect to the pressure, $\mathrm{B}^{\prime}=\mathrm{dB} / \mathrm{dP}$

The computed values of lattice constant (a), bulk modulus (B) and its pressure derivative $\left(\mathrm{B}^{\prime}\right)$ are presented in Table 1 and compared with the experimental [10] and other theoretical results [5$7,9,14-19]$. It is obvious from Table 1 that present results obtained from first principle calculation as well as from the model are in good agreement with experimental and other theoretical results. 
Table 1. Calculated ground state properties of rare earth nitride.

\begin{tabular}{|c|c|c|c|c|}
\hline Crystal & Approximation & $\mathrm{a}[\AA \AA]$ & $\mathrm{B}$ [GPa] & $\mathrm{B}^{\prime}$ \\
\hline \multirow[t]{9}{*}{$\mathrm{CeN}$} & Present & 5.020 & 154 & 3.5 \\
\hline & Expt. & $5.021(2)^{\mathrm{a}}$ & $156(3)^{\mathrm{a}}$ & $4.0^{\mathrm{a}}$ \\
\hline & Others & $5.024^{\mathrm{a}}$ & $158.1^{\mathrm{a}}$ & $3.3^{\mathrm{a}}$ \\
\hline & & $5.021(1)^{\mathrm{b}}$ & $153(1)^{\mathrm{h}}$ & - \\
\hline & & $5.020(1)^{\mathrm{c}}$ & $159.0^{\mathrm{i}}$ & $3.3^{\mathrm{k}}$ \\
\hline & & $5.023(1)^{\mathrm{d}}$ & $140.2^{\mathrm{j}}$ & \\
\hline & & $5.020(1)^{\mathrm{e}}$ & $158.1^{\mathrm{k}}$ & \\
\hline & & $5.022(2)^{\mathrm{f}}$ & & \\
\hline & & $5.019(2)^{\mathrm{g}}$ & & \\
\hline
\end{tabular}

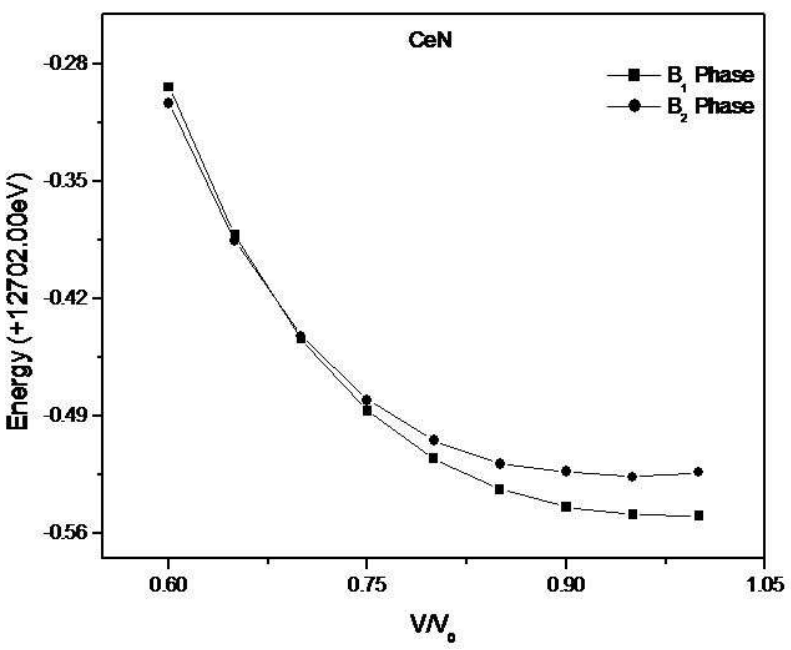

Fig. 1. Variation of total energy with relative volume of the $\mathrm{B} 1$ and $\mathrm{B} 2$ phases in $\mathrm{CeN}$.

\subsection{Structural properties}

The electronic band structures BS calculations have been performed to estimate the total energy of cerium nitride by using the the plane-wave pseudopotential density functional theory. The total energies are plotted against different compressions for the present compound in Fig. 1. The minimum of the curves defines the equilibrium volume $\mathrm{V}_{0}$. The calculation of phase transition pressure has been carried out by estimating enthalpy in both phases of the structures. At a particular pressure these two phases coexist. The variation of enthalpy with pressure has been plotted in Fig. 2. The calculated value of phase transition pressure is $68 \mathrm{GPa}$ for the present compound and is comparable with experimental (65 GPa to $70 \mathrm{GPa}$ ) [10] and other theoretical results $(62 \mathrm{GPa}, 88 \mathrm{GPa}$, $68 \mathrm{GPa})[6,7,10]$ shown in Table 2 . At the phase transition pressure a sudden collapse in volume takes place at the transition pressure. The discontinuity in volume $\left(\Delta \mathrm{V} / \mathrm{V}_{0}\right)$ at the transition pressure is obtained from the phase diagram. The compression curve for $\mathrm{CeN}$ is plotted in Fig. 3.

\subsection{Elastic properties}

Elastic properties of materials are closely related to many fundamental solid-state properties, such as specific heat, thermal expansion coefficient and Debye temperature, etc. Cubic crystals have three independent elastic constants, namely $\mathrm{C}_{11}$,

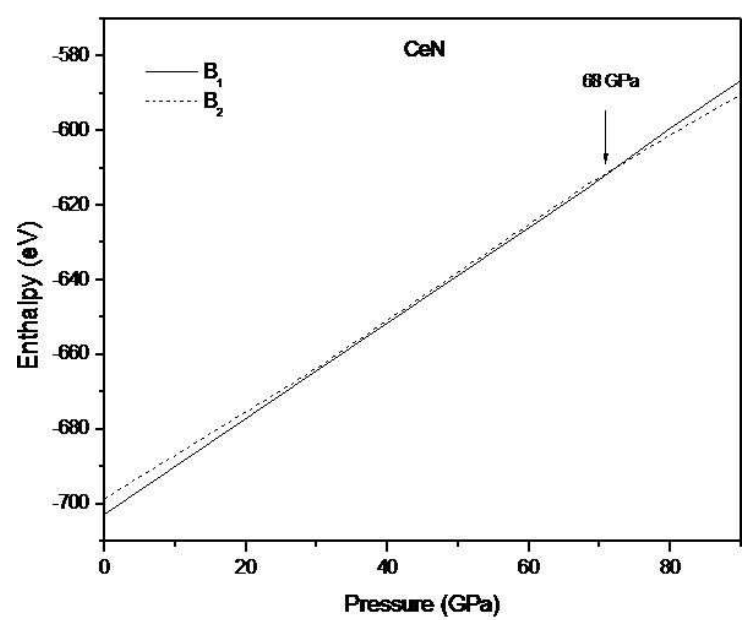

Fig. 2. Variation of enthalpy $(\mathrm{H})$ with pressure for $\mathrm{CeN}$.

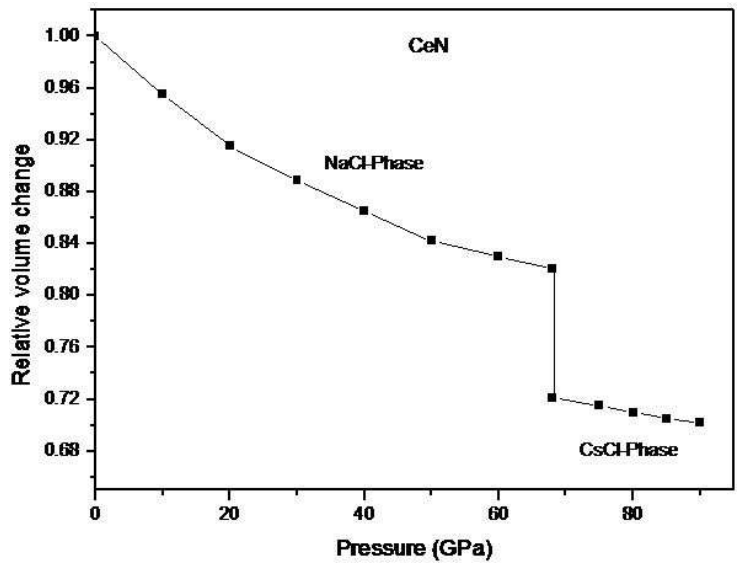

Fig. 3. Relative volume versus pressure for $\mathrm{CeN}$. 
$\mathrm{C}_{12}$ and $\mathrm{C}_{44}$. The criterion for mechanical stability of any compound is that the strain energy should be positive, which imposes further restriction on the values of the elastic constants [20] such as:

$$
C_{11}>C_{12} ; C_{44}>0 ; C_{11}+2 C_{12}>0
$$

Table 2. Phase transition and volume change of CeN.

\begin{tabular}{|c|c|c|c|c|c|}
\hline Crystal & \multicolumn{3}{|c|}{ Phase transition pressure } & \multicolumn{2}{|c|}{$\begin{array}{c}\text { Volume change } \\
{[\%]}\end{array}$} \\
\hline \multirow{4}{*}{$\mathrm{CeN}$} & Presen & Others & Expt. & Present & Others Expt. \\
\hline & 68 & $68^{\mathrm{a}}$ & $65-70^{\mathrm{a}}$ & 10.0 & $10.8^{\mathrm{a}}$ \\
\hline & & $62^{\mathrm{b}}$ & & $5^{b}$ & $10.9^{\mathrm{a}}$ \\
\hline & & $88^{c}$ & & $5.8^{\mathrm{c}}$ & \\
\hline
\end{tabular}

${ }^{\mathrm{a}}[10],{ }^{\mathrm{b}}[6],{ }^{\mathrm{c}}[7]$

These criteria for mechanical stability are satisfied by the calculated elastic constants of $\mathrm{CeN}$ under present study and are reported in Table 3. The Cauchy variation $\left(\mathrm{C}_{12}-\mathrm{C}_{44}\right)$ has also been reported in this Table. Its value is negative for $\mathrm{CeN}$.

The shear modulus $G$ is one of several quantities for measuring the stiffness of materials. All of them arise in the generalized Hooke's law. These quantities are Young modulus $\mathrm{Y}$ and bulk modulus B. The shear modulus $\mathrm{G}$ can be defined by the following equations:

$$
G=\left(G_{V}+G_{R}\right) / 2
$$

where $\mathrm{G}_{\mathrm{V}}=\left(2 \mathrm{C}+3 \mathrm{C}_{44}\right) / 5$

$$
G_{R}=15\left(6 / C+9 / C_{44}\right)^{-1}
$$

where $\mathrm{C}=\left(\mathrm{C}_{11}-\mathrm{C}_{12}\right) / 2, \mathrm{G}_{\mathrm{V}}$ is the Voigt shear modulus and $G_{R}$ is the Reuss shear modulus.

The Young modulus $\mathrm{Y}$ can be calculated from the bulk modulus B and the shear modulus $G$ by the following equation:

$$
Y=9 B G /(3 B+G)
$$

In most applications of elasticity theory for the problems in petroleum geophysics, the elastic medium is assumed to be isotropic. On the other hand, most crustal rocks are found experimentally to be anisotropic. A material is said to be anisotropic if the value of a vector measurement of a rock property varies with direction. We have calculated the elastic anisotropic parameter and Poisson ratio $(\sigma)$ of present compound, using the following relations:

$$
\begin{gathered}
A=\frac{2 C_{44}}{C_{11}-C_{12}} \\
\sigma=\frac{3 B-2 G}{6 B+2 G}
\end{gathered}
$$

The calculated values of shear modulus, Young modulus, anisotropy parameter and Poisson ratio are given in Table 3 . These results have been compared with others theoretical results [7]. Other results have been obtained using two-body potential theory with ionic modified charge. They have used only the coulombic and van der Waals interaction and overlap repulsive interaction, ignoring covalency effect. There are no experimental results available for elastic moduli of CeN. The brittle/ductile behavior of $\mathrm{CeN}$ is also determined with ratio of $\mathrm{B} / \mathrm{G}$. The present value of $\mathrm{B} / \mathrm{G}$ is 1.666 . This value is much lower than the critical value of 1.75. According to Pugh [21], a material is ductile if $\mathrm{B} / \mathrm{G}>1.75$. From Table 3 , it can be observed that the present nitride is brittle in nature.

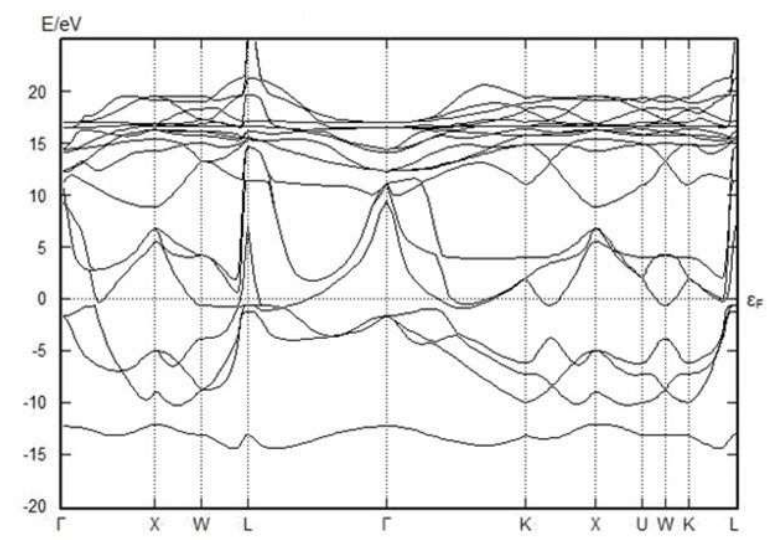

Fig. 4. Electronic band structure (BS) of CeN.

\subsection{Electronic properties}

To study the electronic properties of $\mathrm{CeN}$, the band structure, total and partial density of states 
Table 3. Calculated elastic properties of CeN.

\begin{tabular}{ccccccccccc}
\hline Crystal & Approximation & $\mathrm{C}_{11}$ & $\mathrm{C}_{12}$ & $\mathrm{C}_{44}$ & $\mathrm{C}_{12}-\mathrm{C}_{44}$ & $\mathrm{G}$ & $\mathrm{Y}$ & $\mathrm{A}$ & $\sigma$ & $\mathrm{B} / \mathrm{G}$ \\
\hline \hline $\mathrm{CeN}$ & Present & 315.0 & 73.5 & 77.2 & -3.7 & 92.41 & 231.02 & 0.6393 & 0.249 & 1.666 \\
(Others) & $299.0^{\mathrm{a}}$ & $60.8^{\mathrm{a}}$ & $63.0^{\mathrm{a}}$ & $-2.2^{\mathrm{a} *}$ & $119.1^{\mathrm{a}}$ & $278.45^{\mathrm{a}}$ & - & $0.168^{\mathrm{a}}$ & - & \\
\hline
\end{tabular}

a [7], ${ }^{*}$ Calculated from others theoretical results.

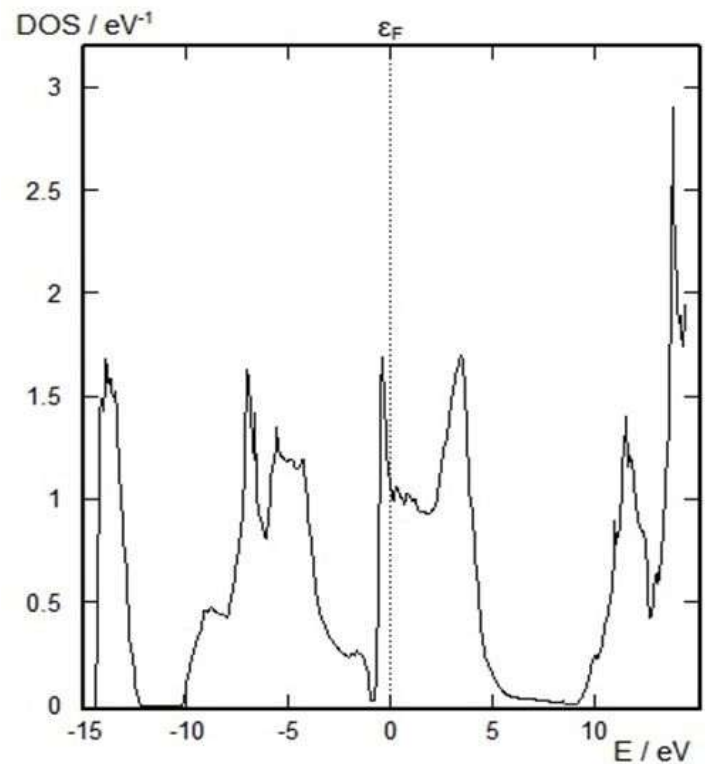

Fig. 5. Total density of states of $\mathrm{CeN}$ in $\mathrm{NaCl}$ phase.

has been reported. Fig. 4 represents the calculated electronic band structure (BS) along the principle symmetry direction for $\mathrm{CeN}$ in its rock-salt (B1) phase under ambient conditions. The band structure of CeN shows an indirect overlap of the p-band at $\Gamma$ with the d-band at $X$. It is clear from this figure that $\mathrm{p}-\mathrm{d}$ band structure is different from the standard $\mathrm{ScN}$-like compounds. The Fermi level is set to zero energy. It can be seen that there are some bands crossing the Fermi level which indicates the metallic behavior of $\mathrm{B} 1$ phase of the $\mathrm{CeN}$. In order to understand the elementary contribution of all the atoms to the electronic structure of present compound we have studied total density of states (TDOS) in its rock-salt (B1) phase at ambient pressure. The TDOS of $\mathrm{CeN}$ is presented in Fig. 5. It is obviously seen from Fig. 5 that in TDOS, almost all the $f$-electron peaks are located in the region of -1.0 to $4.0 \mathrm{eV}$. These peaks arise due to p-state, which is clearly seen in Fig. 6, presenting
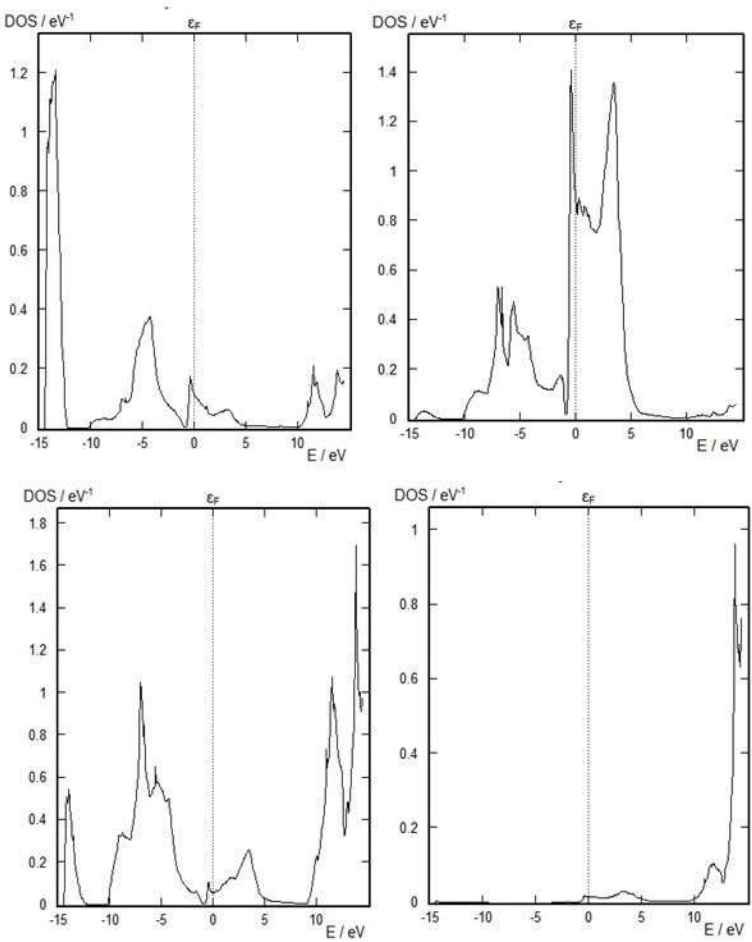

Fig. 6. Partial density of states (PDOS) in the $\mathrm{NaCl}$ phase of CeN in $s, p, d$ and $f$ states.

the partial density of states (PDOS) of CeN for s, $p, d$ and $f$ states, respectively.

\section{Conclusions}

In the present study, the first principle calculations have been used to investigate the structural and electronic properties of $\mathrm{CeN}$. The calculated ground state properties are in good agreement with experimental and other theoretical results. The value of pressure induced B1-B2 transformation obtained in the calculations is 68 GPa with the volume collapse of $10.0 \%$, which experimentally occurs in the range of $65 \mathrm{GPa}$ to $70 \mathrm{GPa}$ with the volume collapse of $10.9 \%$. 
These results are also in good agreement with the calculated values. The elastic constants and modulus of elasticity are also in fair agreement with available results. The present rare earth nitride shows brittle behavior while its B1 phase shows metallic behavior.

\section{Acknowledgements}

The authors are thankful to the Department of Science \& Technology (DST), New Delhi, for the financial support to this work. One of the authors (PB) is grateful to the Department of Science \& Technology (DST), New Delhi, for awarding WOS'A' (Grant No. SR/WOS-A/PS-17/2013).

\section{References}

[1] Larson P., Lambrecht W.R.L, Chantis A., SchilfgaArde van M., Phys. Rev. B, 75 (2007), 045114.

[2] Preston A.R.H., Granvile S., Housden D.H., Ludbrook B., Ruck B.J., Trodahl H.J., Phys. Rev. $B, 76$ (2007), 245120.

[3] Mei A.B., Rockett A., Hultman L., Petrov I., Greene J.E., J. Appl. Phys., 114 (2013), 193708.

[4] Duan C.G., Sabirianov R.F., Mei W.N., Dowben P.A., Jaswal S.S., Tsymbal E.Y., J. Phys. Condens. Mat., 19 (2007), 315220.

[5] Danan J., Novion de C., Lallement R., Solid State Commun., 7 (1969), 1103.

[6] Svane A., Szotek Z., Temmermen W.M., Laqsqaard J., Winter H., J. Phys.-Condens. Mat., 10 (1998), 5309.

[7] Rukmangad A., Anayas M., Sanyal S.P., Indian J. Pure Ap. Phy., 47 (2009), 114.
[8] LeE T.Y., Gall D., Shin C.S., Hellgren N., Petrov I., Greene J.E., J. Appl. Phys., 94 (2003), 921.

[9] Kanchana V., Vaitheeswaran G., Zhang X., Ma Y., Svane A., Eriksson O., Phys. Rev. B, 84 (2011), 205135.

[10] Olsen J.S., JŘrgenSEN J.-E., GerWARd L., Vaitheeswaran G., Kanchana V., Svane A., $J$. Alloy. Compd., 533 (2012), 29.

[11] Baroni S., CARso Dal A., Gironcoli S.D., GianNOZZI P., J. Phys.-Condens. Mat., 21 (2009), 395502.

[12] Giannozzi P., Cavazzoni C., Il Nouvo Cimento C, 32 (2009), 49.

[13] Murnaghan F.D., P. Natl. Acad. Sci. USA, 30 (1944), 244.

[14] Iandelli A., Botti E., Il Nouvo Cimento $C, 25$ (1937), 129.

[15] Gambino R.J., Cuomo J.J., J. Electrochem. Soc., 133 (1966), 401.

[16] Brown R.C., Clark N.J., Mater. Res. Bull., 9 (1974), 1007.

[17] Olcese G.L., J. Phys. F-Met. Phy., 9 (1979), 569.

[18] Schlegel A., Kaldis E., Wachter P., Zurcher C., Phys. Lett. A, 66 (1978), 125.

[19] JAKOBSEN J.M., MAdSEN G.K.H., JŘRGENSEN J.-E., Olsen J.S., Gerward L., Solid State Commun., 121 (2002), 447.

[20] Mattesini M., Soler J.M., Yndurain F., Phys. Rev. B, 73 (2006), 094111.

[21] Pugh S.F., Philos. Mag., 45 (1954), 823. 